\title{
Theoretical study of the effects of different substituents of tetrathiafulvalene derivatives on charge transport
}

\author{
LI HuiXue ${ }^{1,2 *}$, WANG XiaoFeng ${ }^{1,2} \&$ LI ZhiFeng ${ }^{1,2}$ \\ ${ }^{1}$ College of Life Science and Chemistry, Tianshui Normal University, Tianshui 741001, China; \\ ${ }^{2}$ Key Laboratory for New Molecule Design and Function of Gansu Education Department, Tianshui 741001, China
}

Received January 12, 2012; accepted March 25, 2012; published online May 15, 2012

\begin{abstract}
Density functional theory calculations were carried out to investigate the charge transfer of four tetrathiafulvalene derivatives. Perfluorination of dibenzo-tetrathiafulvalene (DB-TTF) increased the reorganization energy and was considered disadvantageous for the charge-transport process. Fluorination lowered the frontier orbitals of the compound, favoring electron-rather than hole-transport due to the low injection barrier. While intra-ring substitution of carbons of benzene with $\mathrm{N}$ atoms did not increase the reorganization energy, it enforced thermodynamic stability and decreased the charge injection barrier due to lowering the frontier orbital. Calculation results also showed that introduction of $\mathrm{NH}_{2}$ to DB-TTF can change the crystal structure and charge mobility, thus providing a method with which to promote $\pi$-stacked structures. Calculation of charge transfer integrals using site energy correction methods was found to be more suitable for perfluorinated DB-TTF because it exhibits remarkable polarization effects.
\end{abstract}

theoretical study, substituting effect, charge transport

Citation: $\quad$ Li H X, Wang X F, Li Z F. Theoretical study of the effects of different substituents of tetrathiafulvalene derivatives on charge transport. Chin Sci Bull, 2012, 57: 4049-4056, doi: 10.1007/s11434-012-5222-z

Organic charge transfer materials have recently gained significant interest due to their low cost, easy chemical modification, and structural flexibility [1-5]. Charge carrier mobility is a measure of the quality of organic semiconductors and a primary factor that determines the performance of organic field effect transistors (OFETs) [6-9]. Excellent charge carrier mobilities, which are of the same order as amorphous silicon ( 0.1 to $\left.1.0 \mathrm{~cm}^{2} /(\mathrm{V} \mathrm{s})\right)$, have been achieved in OFETs through the unremitting efforts of many scientists [4,10-12]. Tetrathiafulvalene (TTF) derivatives have received increased attention because these compounds exhibit various intra- and intermolecular interactions, including van der Waals interactions, weak hydrogen bonding, $\pi-\pi$ stacking, and sulfur-sulfur interaction [11,13,14], all of which decide the crystal structures involved and consequentially affect the electron coupling between adjacent molecules. TTF derivatives are generally soluble in various sol-

*Corresponding author (email: li_hx2001@126.com) vents, which permit the use of inexpensive deposition techniques, such as spin-coating and inkjet printing, to obtain them. Furthermore, the electronic and structural characteristics of these heterocyclic compounds can be modified by introduction of different types of substituents at the 2, 3, 6, and 7 positions of the TTF core [15]. Many OFET-based TTF derivatives have been obtained using vacuum techniques and solution, and several aromatic rings, such as benzene, naphthalene, and thiophene rings have been introduced to TTF. These derivatives exhibit excellent charge transfer properties, the highest charge mobility of which was reported to be $3.6 \mathrm{~cm}^{2} /(\mathrm{V}$ s) [16]. Mas-Torrent et al. $[17,18]$ studied dithiophene-tetrathiafulvalene (DT-TTF) and dibenzo-tetrathiafulvalene (DB-TTF), which contain similar molecules but present different crystal packing types, and found that different intermolecular interactions significantly influence the electronic transport properties of the compounds. Naraso et al. [19] found that OFET devices based on halogeno-substituted quinoxaline rings fused with 
TTF show excellent n-type performance and high carrier mobility. To date, most available organic semiconductors involve hole-transporting (p-type) materials, and reports on n-type semiconductors are relatively rare. The lack of studies on such n-type materials has been a significant impediment to applications using these organic-based devices. Therefore, determination of new molecules that are able to act as organic semiconductors, especially n-type materials, is of great importance.

The lowest unoccupied molecular orbital (LUMO) level of a semiconducting molecule must be lowered (i.e., its electron affinity must be increased) substantially to align with the work function of the metal for the fabrication of n-channel transistors with the same electrodes as those used for p-channel transistors, such as gold, which is the most common electrode known today due to its environmental stability [4]. This process is achieved by taking a known semiconducting core molecule and adding strong electronwithdrawing groups, such as fluorine $(\mathrm{F})$, cyano, or diimide moieties [2-4,20]. In this paper, we investigate the influence of two electron-withdrawing groups ( $\mathrm{F}$ and $\mathrm{N}$ atoms) and one electron-donating group (amino, $\mathrm{NH}_{2}$ ) on the charge transfer properties of DB-TTF.

\section{Computational approaches}

The incoherent hopping model is adopted to illustrate the charge transfer between a neutral molecule and a neighboring charged molecule in organic materials. The basic assumption here is that charge transfer is a slow process such that molecules have enough time to reach equilibrium. Within the hopping description used in this work, the charge transfer can be characterized by a diffusion process, and the carrier mobility (eq. (1)) can be well described by the Einstein relation $[9,21]$ :

$$
\mu=e D / k_{\mathrm{B}} T,
$$

where $\mu$ is the drift charge transfer mobility, $e$ is the electron charge, $k_{\mathrm{B}}$ is the Boltzmann constant, $T$ is the temperature and $D$ is the charge diffusion coefficient, which can be approximately evaluated as [8]

$$
D=\frac{1}{2 d} \sum_{i} r_{i}^{2} k_{i} p_{i}
$$

where $i$ is a specific transfer pathway, $r_{i}$ is the transfer distance (intermolecular center to center distance) that runs over all nearest adjacent molecules, $1 / k_{i}$ is the transfer time, and $d$ is the spatial dimension, which is equal to 3 for the crystal. $P_{i}$ is the relative probability for the $i$ th pathway, which is given by

$$
P_{i}=k_{i} / \sum_{i} k_{i}
$$

Determination of charge transfer rates between molecular dimers is necessary as the above simulation is employed. The rate constant can be modeled by classical Marcus theory when the charge transfer in an adjacent molecular dimer is assumed to be a self-exchange reaction process, which is represented as follows [22,23]

$$
k_{\mathrm{et}}=\frac{V^{2}}{\hbar}\left(\frac{\pi}{\lambda_{ \pm} k_{\mathrm{B}} T}\right)^{1 / 2} \exp \left(-\frac{\lambda_{ \pm}}{4 k_{\mathrm{B}} T}\right),
$$

where $V$ is the transfer integral, $\lambda_{ \pm}$is the reorganization energy.

Reorganization energy and transfer integral are two key factors that influence the charge transfer rate based on eq. (4). Several studies have described how to obtain these parameters from first-principles calculations [9-11,24]. Reorganization energy has both internal and external contributions, with the internal contribution arising from geometric changes in the geometry of the molecular dimer when electron transfer takes place, and the external contribution coming from changes in the surrounding media accompanying the charge transfer. Moderate contributions to the relaxation energy of organic solids and weak polar media are in the order of a few tenths of an electronvolt [25,26]; thus, external contributions are often neglected and only internal contributions are taken into account in organic crystals.

The internal reorganization energy can be obtained using the adiabatic potential energy surface method [27,28], which considers the sum of two relaxation energy terms: (i) the energy difference of the neutral molecule in the optimal charged geometry and in the equilibrium neutral geometry, and (ii) the energy difference of the charged molecule in these two geometries. The reorganization energies for hole transport $\left(\lambda_{+}\right)$and electron transport $\left(\lambda_{-}\right)$are calculated from positively and negatively-charged molecules, respectively.

Several methods have been proposed to evaluate the transfer integral within a molecular dimer. A simple and widely used method for calculating the charge transfer integral is Koopmans's theorem (KT) approximation, which estimates $V$ as half the splitting of the highest occupied molecular orbital (HOMO) or Lowest unoccupied molecular orbital (LUMO) levels in a neutral dimer for hole (electron) transfer [29]. The KT approximation is reliable when the diabatic energies of the donor and acceptor states are the same (i.e., in a symmetric dimer, or more generally, in the transition state structure). Otherwise, KT approximation can overestimate the transfer integral considerably when the molecules are not equal in symmetry [8,30].

A more direct and simpler way of calculating the transfer integral involves direct evaluation of the coupling element between frontier orbitals using the unperturbed density matrix of the dimer Fock operator. In this case, the transfer integral is calculated using the PW91PW91/6-31G* basis set, which has been proven to be simple, efficient, and reliable [31,32]. The electronic coupling for hole or electron transfers in the direct scheme can be written as 


$$
V=\left\langle\phi_{\text {HOMO/LUMO }}^{0 \text {,site1 }}\left|F_{0}\right| \phi_{\text {HOMO/LUMO }}^{0, \text { site2 }}\right\rangle,
$$

where $\phi_{\mathrm{HOMO} / \mathrm{LUMO}}^{0 \text {,site }}$ and $\phi_{\mathrm{HOMO} / \mathrm{LumO}}^{0 \text {,site2 }}$ are the HOMO or LUMO of isolated molecules 1 and 2, respectively, among neighboring molecules, and $F_{0}$ is the Fock operator for the dimer for a fixed pathway; the suffix zero indicates that the molecular orbitals appearing in the operator (the density matrix, for instance) are unperturbed. $F_{0}=S C \varepsilon C^{-1}$ is the Fock operator, where $S$ is the overlap matrix for the dimer taken from the crystal structure, and the Kohn-Sham orbital coefficient $C$ and eigenvalue $\varepsilon$ can be obtained from one-step diagonalization without interaction, which can be performed in the Gaussian 03 package [33]. The direct coupling method (DC) used in this work has much less computational cost than other methods because there is no need to construct the dimer Hamiltonian or obtain energy levels.

The energies of the individual orbitals in the dimer, called site energies, are included in estimating the effective electronic coupling to take the polarization effects explicitly into account for an asymmetric dimer. Senthilkumar et al. [34] and Edward et al. [35] proposed the site energy correction (SEC) model, where the site energy $H_{i i}$ and charge transfer integral $V$ are expressed as

$$
\begin{gathered}
H_{11(22)}=\frac{\left(h_{11}+h_{22}\right)-2 h_{12} S_{12} \pm\left(h_{11}-h_{22}\right) \sqrt{1-S_{12}^{2}}}{2\left(1-S_{12}^{2}\right)}, \\
V=\frac{h_{12}-\frac{1}{2}\left(h_{11}+h_{22}\right) S_{12}}{1-S_{12}^{2}},
\end{gathered}
$$

where $h_{i j}=\left\langle\phi_{i}\left|h_{K S}\right| \phi_{j}\right\rangle$ and $S_{i j}=\left\langle\phi_{i}|S| \phi_{j}\right\rangle$.

\section{Results and discussion}

The structures of the compounds studied in this paper are shown in Figure 1.

\subsection{Crystal packing}

Charge transport properties critically depend on the relative orientations and solid-state packing of the species involved.

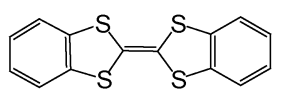

DB-TTF

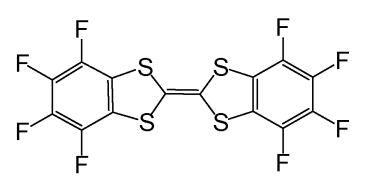

OFDB-TTF

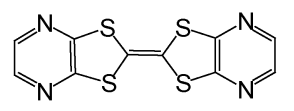

DP-TTF

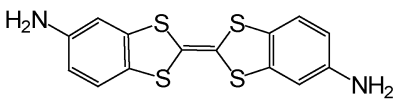

$\mathrm{NH}_{2}$-DB-TTF
Figure 1 Molecular structures of TTF derivatives ( $\mathrm{H}$ atoms were omitted for simplicity).
The packing of a molecular crystal is a compromise that involves all of the intermolecular interactions between its molecules [36]. Strong interactions can change the optimum energy conformation in a substituted molecule compared with that in an isolated molecule. Consequently the geometrical distribution of molecules in the packing is a minimum of the total interaction energy. Therefore, knowing the strength of the intermolecular interactions is very helpful to describe the packing. Dominant interactions are traditionally identified by looking at the shortest intermolecular contacts present in the crystal. TTF-based materials exhibit various intra- and intermolecular interactions, such as van der Waals interactions, $\pi-\pi$ stacking, and sulfur-sulfur interactions. The neutral TTF molecule at the B3LYP level shows a boat conformation, which is due to the fact that the average angle of a planar five-membered ring must be $108^{\circ}$. However, the $\mathrm{C}-\mathrm{S}-\mathrm{C}$ bond angle is usually $92^{\circ}$; thus, a boat conformation is necessary to decrease strain and to obtain a small bond angle. But the most TTF-based neutral molecules exhibit quasi-planar conformations in their crystal packing due to intra- and intermolecular interactions.

The crystal structures of DB-TTF present a herringbone arrangement; it has a monoclinic unit cell with two molecules, and the crystal structure shows that the molecules have more or less edge-to-face packing. The molecules form a zigzag chain along the $c$ axis and the shortest contact between two sulfur atoms of neighboring molecules is 0.375 nm. In contrast to DB-TTF, DP-TTF presents a triclinic system with a unit cell containing two molecules; its crystal structure also shows a herringbone motif, where the zigzag chain of the molecules is along the $a$ axis and the shortest distance of sulfur-sulfur atoms from adjacent molecules is $0.381 \mathrm{~nm}$. The unit cell of OFDB-TTF belongs to the triclinic system with $\mathrm{P}_{1}$ space group and contains two molecules; the shortest contacting of $\mathrm{S} \cdots \mathrm{S}$ is $0.380 \mathrm{~nm}$. $\mathrm{NH}_{2}$-DB-TTF has a monoclinic unit cell with two molecules and exhibits face-to-face $\pi$ - $\pi$ stacking, where the interplanar distance is $0.389 \mathrm{~nm}$. The stacking manner is obviously different from the other compounds and the shortest contact between $\mathrm{S} \cdots \mathrm{S}$ is $0.379 \mathrm{~nm}$ from the adjacent dimer. Moreover, all of the atoms of $\mathrm{NH}_{2}$-DB-TTF are in the same plane. All the crystal structures and charge hopping pathways are shown in Figure 2.

\subsection{Frontier orbital energy level and reorganization energies}

A typical OFET is composed of a gate electrode, dielectric layer, organic semiconductor layer, and source-drain (S-D) electrodes. Carrier injection from the S-D electrode into the organic layer mainly depends on the barrier between the work function of the metal electrode and the HOMO or LUMO energy level of the organic semiconductors. P-type organic semiconductors typically have HOMO levels between -4.9 and $-5.5 \mathrm{eV}$, resulting in ohmic contact with 


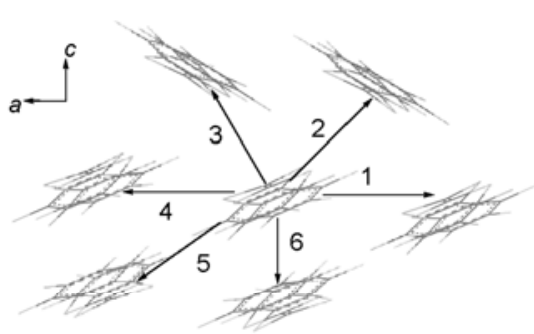

OFDB-TTF a
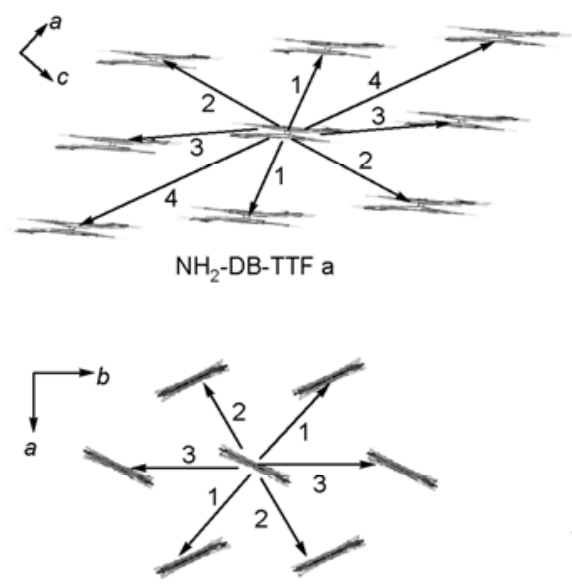

DB-TTF a

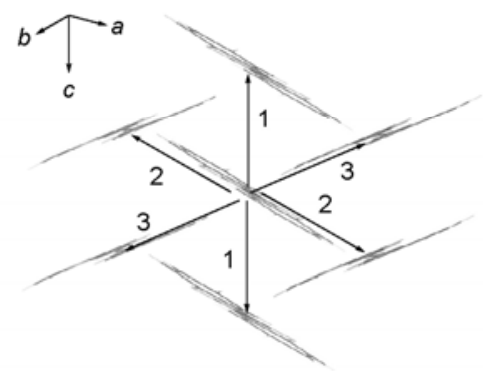

DB-TTF a
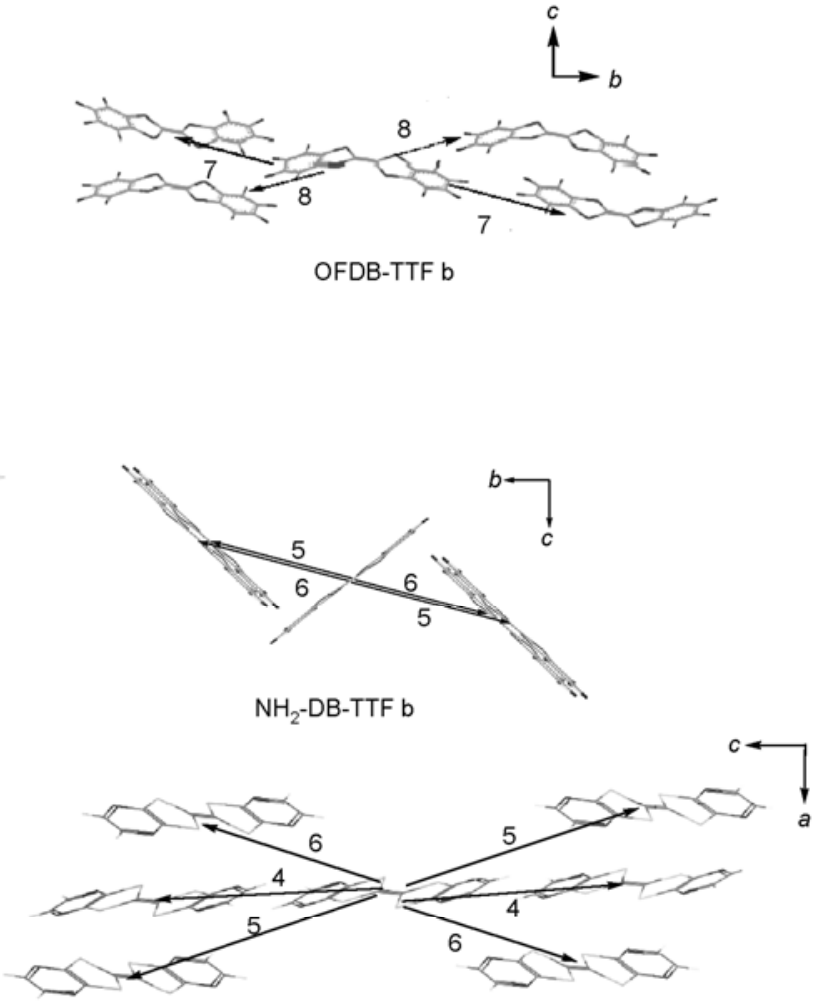

DB-TTF b

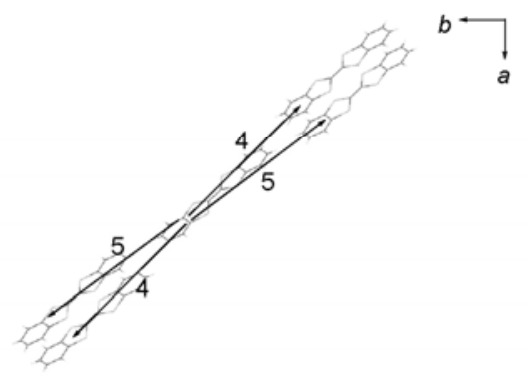

DB-TTF b

Figure 2 Charge hopping pathway schemes for TTFs.

high work-function metals, such as gold $(5.1 \mathrm{eV})$ and platinum $(5.6 \mathrm{eV})$. N-type materials, on the other hand, typically have LUMO levels between -3 and $-4 \mathrm{eV}$ and have better contact with low work-function metals, such as calcium and lithium. However, despite its high work function, gold has been observed to typically form good top contacts with organic n-type materials [37]. The frontier orbital levels of TTF derivatives are listed in Table 1.

When two hydrogen atoms in DB-TTF are substituted with the electron-donating groups $\mathrm{NH}_{2}$ to form $\mathrm{NH}_{2}$-DBTTF, the HOMO and LUMO energy levels of the compound increase and its energy gap decreases compared with that of DB-TTF. The HOMO of $\mathrm{NH}_{2}$-DB-TTF becomes $-4.218 \mathrm{eV}$ while that of DB-TTF is $-4.701 \mathrm{eV}$. As well, the LUMO of $\mathrm{NH}_{2}$-DB-TTF increases from $-1.123 \mathrm{eV}$ of DB-TTF to $-0.762 \mathrm{eV}$. Potential barriers are very high when charge carriers are injected into this semiconductor from an Au electrode, so $\mathrm{NH}_{2}$-DB-TTF may not be a good charge transporting material. Hydrogen bonds are present between molecules of $\mathrm{NH}_{2}$-DB-TTF for the introduction of amino groups. The shortest distance between $\mathrm{N}-\mathrm{H} \cdots \mathrm{N}$ is $0.260 \mathrm{~nm}$, implying that electrostatic interaction is dominant and that the nature of crystal packing is face-to-face $\pi$ stacking instead of a herringbone arrangement.

$\mathrm{N}$-type materials are typically created by attachment of strong electron-withdrawing groups, such as F, cyano, and alkanoyls [38], to the compound of interest, thus lowering the LUMO energy level and decreasing sensitivity to ambient water and oxidation. DP-TTF is a compound in which pyrazine rings replace two benzene rings. Its frontier orbital energy levels decrease due to electron-withdrawing effects, improving charge injection from metal electrodes compared 
Table 1 HOMO and LUMO energies, HOMO-LUMO gaps $\left(E_{\text {gap }}\right)$, and reorganization energies obtained by adiabatic potential methods using B3LYP/6$31 \mathrm{G}(\mathrm{d})$

\begin{tabular}{lccccc}
\hline \multirow{2}{*}{ Molecule } & HOMO (eV) & \multirow{2}{*}{ LUMO (eV) } & $E_{\text {gap }}(\mathrm{eV})$ & \multicolumn{2}{c}{ Reorganization energy (eV) } \\
\cline { 4 - 6 } & & -1.123 & 3.578 & 0.250 & $\lambda_{+}$ \\
\hline DB-TTF (planar) & -4.701 & -1.321 & 3.508 & 0.250 & 0.412 \\
DP-TTF (planar) & -4.829 & -1.796 & 3.837 & 0.478 & 0.287 \\
OFDB-TTF (boat) & -5.633 & -0.762 & 3.456 & 0.239 & 0.692 \\
NH H $_{2}$-DB-TTF (planar) & -4.218 & & &
\end{tabular}

with that observed from DB-TTF. Such a process implies that DP-TTF is also more stable in air. DB-TTF turns into OFDB-TTF when all of its hydrogen atoms are substituted with $\mathrm{F}$ atoms. In this case, the LUMO energy level decreases from -1.123 to $-1.796 \mathrm{eV}$ and the HOMO energy level decreases from -4.701 to $-5.633 \mathrm{eV}$. Obviously, OFDBTTF possesses the smallest barrier in all TTF derivatives when gold electrodes are used.

The reorganization energy must be minimized to achieve high charge carrier mobility based on Marcus theory, which means that molecular configuration transformations must be minimized while charges transfer. The OFDB-TTF molecule shows a boat-configuration both in its crystal and in the calculation at the B3LYP/6-31G* level. In comparison, DB-TTF, DP-TTF, and $\mathrm{NH}_{2}$-DB-TTF are quasi-planar in their crystalline packing, which indicates that OFDB-TTF undergoes more configuration deformations and presents higher reorganization energies when charge carriers pass through it. Calculations of pentacene, sexithiophene, and their perfluorinated analogues showed that perfluorination increases the reorganization energy of the charge-transport process, which originates from additional contributions of C-F bond stretching [5]. The $\lambda_{+}$and $\lambda_{-}$of OFDB-TTF are 0.478 and $0.692 \mathrm{eV}$, respectively, the largest in all of the compounds studied in this work. Decreasing reorganization energy to improve material performance is achieved through intra-ring substitution, such as replacement of carbon with other electronegative atoms, which avoids introduction of additional degrees of freedom for geometric relaxation. DP-TTF is a compound with hole and electron reorganization energies that are markedly lowered than those of OFDB-TTF (Table 1).

The $\lambda_{ \pm}$of DB-TTF and $\mathrm{NH}_{2}$-DB-TTF are close to each other and low, indicating that substituent effects are not obvious in them; this finding is attributed to replacement of only two $\mathrm{H}$ atoms in DB-TTF.

\subsection{Charge transfer integral and mobility}

The charge transfer integral is another important factor for determining charge mobility. Two different methods, namely, DC and SEC, were employed to calculate the charge transfer integrals of neighboring dimmers through DFT/PW91PW91, and all data are shown in Table 2. The charge transfer integrals of DB-TTF and DP-TTF are close to each other. The largest hole coupling terms $\left(V_{\mathrm{h}}\right)$ for DB-TTF are 88.5 me V from DC and 47.8 me V from SEC, and the largest electron coupling terms $\left(V_{\mathrm{e}}\right)$ are $44.5 \mathrm{me} \mathrm{V}$ from DC and $20.1 \mathrm{me} \mathrm{V}$ from SEC. The largest $V_{\mathrm{h}}$ values for DP-TTF are $19.2 \mathrm{me} \mathrm{V}$ from DC and $21.0 \mathrm{me} \mathrm{V}$ from SEC, and the largest $V_{\mathrm{e}}$ values are $23.3 \mathrm{me} \mathrm{V}$ from $\mathrm{DC}$ and $16.4 \mathrm{me} \mathrm{V}$ from SEC. The calculated values of an identical path using the DC and SEC methods are basically within the same order of magnitude. Overall, the $V_{\mathrm{h}}$ values of both compounds are slightly larger than the $V_{\mathrm{e}}$ values, regardless of whether DC or SEC is used. Considering that the $\lambda_{+}$of these compounds are smaller than $\lambda_{-}$, the calculated hole mobilities of DB-TTF and DP-TTF are higher than the electron mobilities, indicating that both TTF derivatives are p-type materials. The hole mobility of DB-TTF is 0.115 $\mathrm{cm}^{2} /(\mathrm{V} \mathrm{s})$ and the experimental values range from 0.06 to $1.4 \mathrm{~cm}^{2} /(\mathrm{V} \mathrm{s})$. The experimental results are in considerable accordance with the theoretical analysis. Considering the anisotropy of molecule deposition on the substrate, the calculated mobility should adopt a one- or two-dimensional model and the predicted result will increase. DP-TTF also exhibits p-type properties similar to those of DB-TTF, where the hole mobility is larger than the electronic mobility. However, the computed hole mobility $\left(0.025 \mathrm{~cm}^{2} /(\mathrm{V} \mathrm{s})\right)$ of DP-TTF is far greater than the experimental value $\left(3.3 \times 10^{-5} \mathrm{~cm}^{2} /(\mathrm{V} \mathrm{s})\right)$, which implies that there is still ample room for improvement in the experiment.

Introduction of an amino group to DB-TTF to yield $\mathrm{NH}_{2}-$ DB-TTF changes the electronic configuration of the atoms. $\mathrm{NH}_{2}$ can form H-bonds with adjacent molecules, causing alterations to the interactions between molecules. Molecular packing in a unit cell forms a columnar arrangement instead of the herringbone motif in DB-TTF, DP-TTF, and OFDB-TTF crystals. The electron coupling terms obtained from DC and SEC are larger than the hole terms as a whole. This result shows that electrons can be transferred more easily in $\mathrm{NH}_{2}$-DB-TTF and that the calculated electron mobility is larger than the hole mobility.

The packing structure for the dimer along pathway 4 of $\mathrm{NH}_{2}$-DB-TTF, as well as its molecular orbital maps of HOMO and LUMO, are illustrated in Figure 3. Generally, the greater the overlap of the molecular orbitals in the dimer is, the greater the charge transfer integral is; otherwise, the integral decreases between the bonding and antibonding overlaps. For the given dimer, a displacement of about one 
Table 2 Hole $\left(V_{\mathrm{h}}\right)$ and electron $\left(V_{\mathrm{e}}\right)$ transfer integrals of TTF derivatives for different hopping pathways $(r)$ calculated using two different methods by DFT/PW91PW91. Hole $\left(\mu_{+}\right)$and electron $\left(\mu_{-}\right)$mobilities (in $\mathrm{cm}^{2} /(\mathrm{V} \mathrm{s})$ ) are also given ${ }^{\text {a) }}$

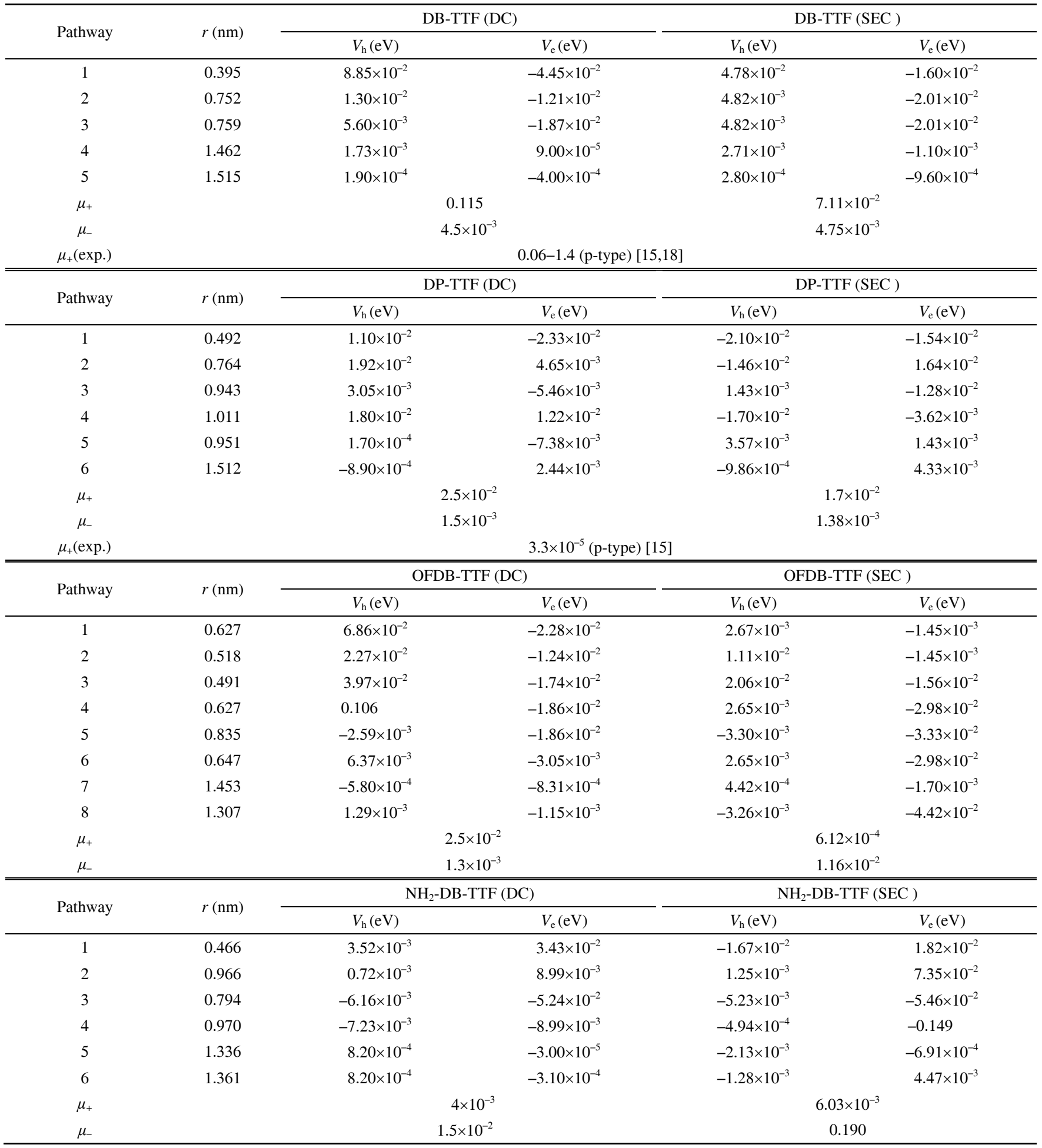

a) $r$ : distance of a charge hop between two adjacent molecules. The temperature was set to $300 \mathrm{~K}$.

phenyl ring width exists. Combined with the HOMO and LUMO orbital charge distributions, the overlap of the LUMO orbital could be deduced to be larger than that of the HOMO, implying easier electron hopping.

OFDB-TTF presents a different scenario where the crystal molecule is a boat conformation instead of planar one, with introduction of eight fluorine atoms. Here, polarization effects between neighboring molecules are more significant. The hole and electron site energies of each dimer were calculated using the DC and SEC methods, and the 


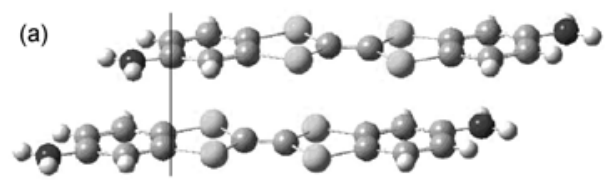

(b)

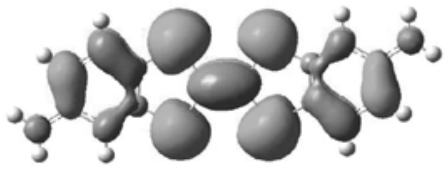

(c)

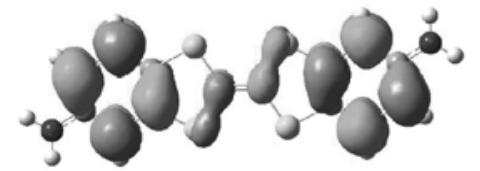

Figure 3 Intermolecular displacement of pathway 4 for $\mathrm{NH}_{2}$-DB-TTF (a) and molecular orbital maps of its HOMO (b) and LUMO (c).

results are shown in Table 3. Due to the nonoccurrence of perturbation between adjacent molecules in DC method, the hole site energy of each pathway is in close proximity to $-4.8 \mathrm{eV}$ and the electron site energies are close to $-2.6 \mathrm{eV}$. Considering the interaction of each dimer in the SEC method, the site energy of each pathway is very different from the results obtained using the DC method, where hole energies range from -4.8 to $-20.2 \mathrm{eV}$, indicating that pathways 1 and 5 are hardly polarized while the others are deviate more or less from the characteristics of a single molecule. The hole transfer integral of pathway 4 is $2.65 \mathrm{meV}$ in the SEC method instead of $106 \mathrm{meV}$ in the DC model, showing remarkable variation. The electron site energies of SEC exhibit larger changes; for example, those of pathways 7 and 8 decrease to $-70.0 \mathrm{eV}$, and the electron transfer integrals accordingly change from 0.831 and $1.15 \mathrm{meV}$ in DC method to 1.70 and $44.2 \mathrm{meV}$ in the SEC method. The data above imply that polarization effects cannot be ignored when calculating charge transfer integrals of OFDB-TTF, and the result obtained means that a study of the molecule using the SEC method may be more appropriate. Calculated SEC results show that OFDB-TTF is an n-type material because its $\mu_{-}\left(0.0116 \mathrm{~cm}^{2} /(\mathrm{V} \mathrm{s})\right)$ is larger than its $\mu_{+}(6.12 \times$ $\left.10^{-4} \mathrm{~cm}^{2} /(\mathrm{V} \mathrm{s})\right)$. However, the semiconductor is a p-type material when the DC method is used, a finding that contradicts the fact that perfluorinated aromatic fused compounds have electronic transmission performance $[2,20,39,40]$. Han group $[41,42]$ pointed out that there exit great difference for the charge transport along different directions in the identical organic semiconductor, they have performed some import calculations about the anisotropic mobility, and the results showed the charge mobility along a specific path can be improved one order of magnitude compared with the total mobility. The study on anisotropy of these TTF derivatives is going on and the results will be reported in another paper.
Table 3 Hole and electron site energies obtained using both the DC and SEC methods for all pathways of OFBD-TTF crystals by DFT/PW91PW91 ${ }^{\text {a) }}$

\begin{tabular}{|c|c|c|c|c|c|}
\hline \multirow[t]{2}{*}{ Pathway } & & \multicolumn{2}{|c|}{$\begin{array}{l}\text { Site energy using the DC } \\
\text { method }\end{array}$} & \multicolumn{2}{|c|}{$\begin{array}{l}\text { Site energy using the SEC } \\
\text { method }\end{array}$} \\
\hline & & Hole $(\mathrm{eV})$ & Electron $(\mathrm{eV})$ & Hole $(\mathrm{eV})$ & Electron $(\mathrm{eV})$ \\
\hline \multirow{2}{*}{1} & $H_{11}$ & -4.816 & -2.585 & -4.894 & -2.624 \\
\hline & $H_{22}$ & -4.816 & -2.585 & -4.894 & -2.624 \\
\hline \multirow{2}{*}{2} & $H_{11}$ & -4.735 & -2.612 & -10.683 & -2.624 \\
\hline & $H_{22}$ & -4.816 & -2.585 & -20.193 & -2.624 \\
\hline \multirow{2}{*}{3} & $H_{11}$ & -4.816 & -2.585 & -11.182 & -2.609 \\
\hline & $H_{22}$ & -4.735 & -2.612 & -17.638 & -2.609 \\
\hline \multirow[b]{2}{*}{4} & $H_{11}$ & -4.816 & -2.585 & -10.437 & -2.580 \\
\hline & $H_{22}$ & -4.816 & -2.585 & -10.408 & -2.608 \\
\hline \multirow[b]{2}{*}{5} & $H_{11}$ & -4.816 & -2.585 & -4.824 & -2.572 \\
\hline & $H_{22}$ & -4.816 & -2.585 & -4.824 & -2.572 \\
\hline \multirow[b]{2}{*}{6} & $H_{11}$ & -4.816 & -2.585 & -10.408 & -2.580 \\
\hline & $H_{22}$ & -4.816 & -2.585 & -10.437 & -2.608 \\
\hline \multirow{2}{*}{7} & $H_{11}$ & -4.735 & -2.612 & -13.142 & -68.288 \\
\hline & $H_{22}$ & -4.816 & -2.585 & -11.883 & -70.211 \\
\hline \multirow{2}{*}{8} & $H_{11}$ & -4.816 & -2.585 & -11.332 & -69.888 \\
\hline & $H_{22}$ & -4.816 & -2.585 & -11.416 & -70.151 \\
\hline
\end{tabular}

a) The Hamilton used in the DC method were unperturbed, whereas that in the SEC method were self-consistent.

\section{Conclusion}

In this work, the rate constants of charge transfer of TTF derivatives were predicted based on the Marcus electron transfer model. A diffusion process simulated charge mobility by Einstein relations. Charge mobility was found to depend mainly on the monomer reorganization energy and the coupling matrix element between dimmers. Both the reorganization energy and transfer integral were calculated at the first-principles DFT level, and results showed that perfluorination increases the stability of radical anions, lowers the injection barrier of electrons, and increases the reorganization energy of the charge-transport process. Moreover, perfluorination causes significant polarization effects. The charge transfer integral was calculated using the SEC method, where the predicted $\mu_{-}$is larger and should be considered as an n-type semiconductor. Introduction of $\mathrm{NH}_{2}$ to DB-TTF changed the distribution of electron density on a single molecule and crystal structure. Consequently, the $\mu_{-}$increased to values larger than those of $\mu_{+}$; frontier orbitals also increased compared with the $\mathrm{NH}_{2}$-free parent compound, implying the presence of a high electron injection barrier and increased instability in air. Predicted results of DB-TTF and DP-TTF are similar to those obtained from experiments using both the DC and SEC methods.

We would like to thank Prof. Qing Shi for providing support and guidance in this work. Some of the calculations were performed on the computer workstation of the Qing Shi group at the Institute of Chemistry, Chinese Academy of Sciences. We would also like to thank Prof. ZhiGang Shuai for providing the code with which we calculated the charge transfer integrals. This work was supported by the Research Fund of Tianshui Normal University for Young College Teachers (TSA1116). 
1 Lan Y K, Huang C I. A theoretical study of the charge transfer behavior of the highly regioregular poly-3-hexylthiophene in the ordered state. J Phys Chem B, 2008, 112: 14857-14862

2 Thomas W, Amsharov K, Ute Z, et al. Organic n-channel transistors based on core-cyanated perylene carboxylic diimide derivatives. J Am Chem Soc, 2008, 130: 4637-4645

3 Newman C R, Frisbie C D, da Silva D A, et al. Introduction to organic thin film transistors and design of n-channel organic semiconductors. Chem Mater, 2004, 16: 4436-4451

4 Zaumseil J, Sirringhaus H. Electron and ambipolar transport in organic field-effect transistors. Chem Rev, 2007, 107: 1296-1323

5 Chen H Y, Chao I. Effect of perfluorination on the charge-transport properties of organic semiconductors: Density functional theory study of perfluorinated pentacene and sexithiophene. Chem Phys Lett, 2005, 401: 539-545

6 Yang X D, Wang L J, Wang C L, et al. Influences of crystal structures and molecular sizes on the charge mobility of organic semiconductors: Oligothiophenes. Chem Mater, 2008, 20: $3205-$ 3211

7 Chisaka J, Lu M, Nagamatsu S, et al. Structure and electrical properties of unsubstituted oligothiophenes end-capped at the betaposition. Chem Mater, 2007, 19: 2694-2701

8 Deng W Q, Goddard W A. Predictions of hole mobilities in oligoacene organic semiconductors from quantum mechanical calculations. J Phys Chem B, 2004, 108: 8614-8621

9 Wang L J, Nan G J, Yang X D, et al. Computational methods for design of organic materials with high charge mobility. Chem Soc Rev, 2010, 39: 423-434

10 Bredas J L, Beljonne D, Coropceanu V, et al. Charge-transfer and energy-transfer processes in pi-conjugated oligomers and polymers: A molecular picture. Chem Rev, 2004, 104: 4971-5003

11 Coropceanu V, Cornil J, da Silva D A, et al. Charge transport in organic semiconductors. Chem Rev, 2007, 107: 926-952

12 Mas-Torrent M, Rovira C. Novel small molecules for organic fieldeffect transistors: Towards processability and high performance. Chem Soc Rev, 2008, 37: 827-838

13 Fourmigué M, Batail P. Activation of hydrogen- and halogenbonding interactions in tetrathiafulvalene-based crystalline molecular conductors. Chem Rev, 2004, 104: 5379-5418

14 Mas-Torrent M, Rovira C. Tetrathiafulvalene derivatives for organic field effect transistors. J Mater Chem, 2006, 16: 433-436

15 Naraso, Nishida J I, Ando S, et al. High-performance organic fieldeffect transistors based on pi-extended tetrathiafulvalene derivatives. J Am Chem Soc, 2005, 127: 10142-10143

16 Leufgen M, Rost O, Gould C, et al. High-mobility tetrathiafulvalene organic field-effect transistors from solution processing. Org Electron, 2008, 9: 1101-1106

17 Mas-Torrent M, Hadley P, Bromley S T, et al. Correlation between crystal structure and mobility in organic field-effect transistors based on single crystals of tetrathiafulvalene derivatives. J Am Chem Soc, 2004, 126: 8546-8553

18 Mas-Torrent M, Hadley P, Bromley S, et al. Single-crystal organic field-effect transistors based on dibenzo-tetrathiafulvalene. Appl Phys Lett, 2005, 86: 012110

19 Naraso, Nishida J, Kumaki D, et al. High performance n- and p-type field-effect transistors based on tetrathiafulvalene derivatives. J Am Chem Soc, 2006, 128: 9598-9599

20 Sakamoto Y, Suzuki T, Kobayashi M, et al. Perfluoropentacene: High-performance p-n junctions and complementary circuits with pentacene. J Am Chem Soc, 2004, 126: 8138-8140

21 Schein L B, McGhie A R. Band-hopping mobility transition in naphthalene and deuterated naphthalene. Phys Rev B, 1979, 20: $1631-1639$
22 Marcus R A. Chemical+electrochemical electron-transfer theory. Annu Rev Phys Chem, 1964, 15: 155-196

23 Marcus R A. Electron-transfer reactions in chemistry-Theory and experiment. Rev Mod Phys, 1993, 65: 599-610

24 Li H X, Zheng R H, Shi Q. Theoretical study on charge carrier mobilities of tetrathiafulvalene derivatives. Phys Chem Chem Phys, 2011, 13: 5642-5650

25 Yin S W, Yi Y P, Li Q X, et al. Balanced carrier transports of electrons and holes in silole-based compounds-A theoretical study. J Phys Chem A, 2006, 110: 7138-7143

26 Nan G J, Wang L J, Yang X D, et al. Charge transfer rates in organic semiconductors beyond first-order perturbation: From weak to strong coupling regimes. J Chem Phys, 2009, 130: 134106

27 Beljonne D, Ye A J, Shuai Z, et al. Chain-length dependence of singlet and triplet exciton formation rates in organic light-emitting diodes. Adv Funct Mater, 2004, 14: 684-692

28 Lemaur V, Da Silva Filho D A, Coropceanu V, et al. Charge transport properties in discotic liquid crystals: A quantum-chemical insight into structure-property relationships. J Am Chem Soc, 2004, 126: 3271-3279

29 Koopmans T. ber die Zuordnung von Wellenfunktionen und Eigenwerten zu den einzelnen Elektronen eines Atoms. Physica, 1934, 1: 104-113

30 Hutchison G, Ratner M, Marks T. Intermolecular charge transfer between heterocyclic oligomers. Effects of heteroatom and molecular packing on hopping transport in organic semiconductors. J Am Chem Soc, 2005, 127: 16866-16881

31 Yang F Y, Chang K J, Hsu M Y, et al. High-performance poly(3hexylthiophene) transistors with thermally cured and photo-cured PVP gate dielectrics. J Mater Chem, 2008, 18: 5927-5932

32 Huang J S, Kertesz M. Intermolecular transfer integrals for organic molecular materials: Can basis set convergence be achieved? Chem Phys Lett, 2004, 390: 110-115

33 Frisch M J, Trucks G W, Schlegel H B, et al. Gaussian 03 E. 01, Pittsburgh PA: Gaussian Inc, 2004

34 Senthilkumar K, Grozema F, Guerra C, et al. Charge transport properties in discotic liquid crystals: A quantum-chemical insight into structure-property relationships. J Am Chem Soc, 2005, 127: 14894-14903

35 Edward F, Valeev V C, Demetrio A. et al. Effect of electronic polarization on charge-transport parameters in molecular organic semiconductors. J Am Chem Soc, 2006, 128: 9882-9886

36 Rovira C, Novoa J J. Strength and directionality of the S $\cdots S$ intermolecular interactions present in TTF-based molecular crystals. A combined statistical and $a b$ initio study. Chem Eur J, 1999, 5: 3689-3697

37 Murphy A, Frechet J. Organic semiconducting oligomers for use in thin film transistors. Chem Rev, 2007, 107: 1066-1096

38 Tang M L, Oh J H, Reichardt A D, et al. Chlorination: A general route toward electron transport in organic semiconductors. J Am Chem Soc, 2009, 131: 3733-3740

39 Heidenhain S B, Sakamoto Y, Suzuki T, et al. Perfluorinated oligo( $p$ phenylene)s: Efficient n-type semiconductors for organic lightemitting diodes. J Am Chem Soc, 2000, 122: 10240-10241

40 Chen H Y, Chao I. Toward the rational design of functionalized pentacenes: Reduction of the impact of functionalization on the reorganization energy. ChemPhysChem, 2006, 7: 2003-2007

41 Huang J D, Wen S H, Deng W Q, et al. Simulation of hole mobility in $\alpha$-oligofuran crystals. J Phys Chem B, 2011, 115: 2140-2147

42 Chai S, Wen S H, Huang J D, et al. Density functional theory study on electron and hole transport properties of organic pentacene derivatives with electron-withdrawing substituent. J Comput Chem, 2011, 32: $3218-3225$

Open Access This article is distributed under the terms of the Creative Commons Attribution License which permits any use, distribution, and reproduction in any medium, provided the original author(s) and source are credited. 